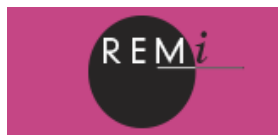

Revue européenne des migrations internationales

vol. 21 - n³ | 2005

Familles, destins personnels et appartenances

collectives en migration

\title{
De la viande halal à l'halal food
}

Comment le halal s'est développé en France?

Florence Bergeaud-Blackler

\section{(2) OpenEdition \\ Journals}

Édition électronique

URL : https://journals.openedition.org/remi/2524

DOI : 10.4000/remi.2524

ISSN : $1777-5418$

Éditeur

Université de Poitiers

Édition imprimée

Date de publication : 1 décembre 2005

Pagination : 125-147

ISBN : 2-911627-45-8

ISSN : 0765-0752

Référence électronique

Florence Bergeaud-Blackler, « De la viande halal à I'halal food », Revue européenne des migrations internationales [En ligne], vol. 21 - n³ | 2005, mis en ligne le 01 décembre 2008, consulté le 14 avril 2022. URL : http://journals.openedition.org/remi/2524 ; DOI : https://doi.org/10.4000/remi.2524

Ce document a été généré automatiquement le 14 avril 2022.

(c) Université de Poitiers 


\title{
De la viande halal à l'halal food
}

\author{
Comment le halal s'est développé en France?
}

Florence Bergeaud-Blackler

Dans les mosquées de France, les questions relatives à la consommation de nourriture et boisson, les manières de table, les rythmes alimentaires sont avec celles du mariage, de la sexualité et des façons de se vêtir parmi les plus souvent posées aux imams, en particulier par les fidèles nés ou scolarisés en France ${ }^{1}$. Parmi ces interrogations revient souvent celle relative à la consommation alimentaire. Un musulman doit-il manger « halal », doit-il s'y astreindre en permanence et partout, dans quel cas peut-il déroger ou surseoir à cette obligation? La réponse la plus courante donnée par les imams est que tout musulman devrait s'approvisionner en viande " halal », abattue selon le rite musulman (Bergeaud-Blackler, 2001). Pourtant une autorité respectée parmi les Musulmans de France, le Cheikh Qaradawi, écrit dans son livre «le licite et l'illicite » (1960) que consommer de la viande produite par les Gens du Livre ${ }^{2}$ est permis. Selon L. T Breze, Président de l'Union des Organisations Islamiques de France, l'opinion émise par le président du CEFR (Conseil Européen pour la Fatwa et la Recherche) signifie qu'en France, pays de "tradition chrétienne », acheter et manger de la viande telle qu'on la trouve dans les boucheries ou les supermarchés est licite pour tout Musulman ${ }^{3}$. Elle se distingue pourtant de celle d'une grande majorité de fidèles musulmans en France pour lesquels la viande halal ne peut être issue que d'un abattage musulman spécifique. Les grandes organisations composant aujourd'hui le Conseil Français du Culte Musulman ne prennent guère de position officielle sur ce thème. Parmi elles, l'UOIF (Union des organisations islamiques de France), considérée aujourd'hui comme l'organisation la plus influente parmi les institutions islamiques en France, est partagée entre l'opinion du Cheikh Qaradawi reprise par l'appareil d'encadrement et celle d'une très grande majorité des membres et sympathisants constituant sa base qui serait plutôt favorable à la mise en place de certification halal spécifique.

2 Cette absence de consensus n'a pas empêché le commerce de viande de boucherie halal de se développer de façon continue depuis le milieu des années quatre-vingt. Offre et consommation de viande halal ont même atteint des sommets dans la seconde moitié des années quatre-vingt-dix (Bergeaud-Blackler, 2000) sans être infléchies par les 
rumeurs de fausse viande halal relayées par la presse ${ }^{4}$ et les acheteurs eux-mêmes. Le climat de «mafia » qui « gangrène » le marché français de la viande sacrifiée, si l'on en croit les enquêteurs de l'hebdomadaire français l'Express ${ }^{5}$, ne semble pourtant pas avoir d'impact significatif sur la confiance des consommateurs. Au contraire, au commerce de viande fraîche s'ajoute désormais celui des produits transformés et la grande distribution ne cache désormais plus sa curiosité gourmande pour le " halal business ».

3 En nous référant à la théorie économique classique, ce climat de défiance (au sens de l'anglais «distrust») devrait conduire vers un effondrement du marché de la viande halal. Les dénonciations de «fausse » viande halal ainsi que les désaccords des religieux sur la nécessité d'une filière spécifique devraient conduire ce marché de la stagnation à l'épuisement. En effet, pour les théoriciens néoclassiques, le développement d'un marché requiert un accord préalable des individus sur les usages et sur la définition du produit échangé (Marshall, 1920 cité par Moureau et Sagot-Duvauroux, 2003). Mais une autre théorie (la théorie des conventions) nous montre que si ces effets d'essoufflement ne se produisent pas toujours, c'est que la qualité d'un produit ne lui pré-existe pas. Au long de son existence, un produit se qualifie, ses qualités sont le fruit de négociations, de conventions entre producteurs, vendeurs, consommateurs et régulateurs formels et informels. Moureau (2003) a montré, à partir du cas du marché de la photographie, que la structure sociale d'un marché se définit en fonction de la façon dont les participants parviennent à un accord sur la définition et les usages du produit, de la nature et la structure des termes de l'échange, le type d'interaction entre les agents, et enfin la définition et la certification de la qualité du produit. Pour la théorie des conventions, toutes ses dimensions, et non l'une d'entre elles, sont à prendre en compte si l'on veut analyser les relations complexes entre échange et propriétés du produit. Les marchés halal ne devraient donc plus être considérés comme des marchés de dupes qui tromperaient d'abord les consommateurs en raison de l'asymétrie des informations entre producteurs/vendeurs et consommateurs. Les morceaux de viandes fraîches halal pourraient être assimilés à des biens « indéterminés » comme ceux que l'on trouve par exemple sur le marché de l'art. Ainsi Lupton (2003) définit le bien de croyance comme un bien pour lequel l'information sur la qualité ne peut être obtenue par le consommateur ni avant ni après l'acquisition du bien. Il n'est ni un bien de recherche dont les qualités sont certifiées par inspection du produit, ni un bien d'expérience, puisque ce n'est pas non plus sa consommation qui rend compte de sa qualitét. La viande halal est un produit indéterminé, un produit "en mal de définition communément admise par tous» (Lupton, 2003: 13). Ceci n'empêche pas pour autant son commerce. Pour la théorie des conventions, ceci n'est qu'un stade de l'existence de tout produit car la qualité d'un produit n'est pas établie une fois pour toutes. «Ainsi quand un produit est considéré comme parfaitement défini, cela signifie en fait qu'il est basé sur des "connaissances stabilisées", mais pas "complètes". Dans ce sens la stabilité du produit est conventionnelle » (Lupton, $2003: 16$ ).

4 S'il est bien difficile de trouver une définition du halal appliquée à l'alimentation, c'est que la «qualité halal» s'est construite progressivement au cours de ces trente dernières années. Cet article propose de décrire et d'analyser cette progression en développant quatre arguments sur la base d'enquêtes menées en Aquitaine sur la production de viandes bouchères halal (viandes bovine et ovine). Ils montrent la grande adaptabilité de ce type de commerce, organisé en petites unités fortement dépendantes de l'état local de l'industrie de la viande, et où les femmes jouent un rôle important dans la définition de la demande. D'ores et déjà, on voit se profiler la mutation 
prochaine des marchés halal. On passe peu à peu d'une conception du halal où l'acte d'égorgement revêt une importance fondamentale (conception héritée des pays d'origines) à une conception où halal devient synonyme de pureté et ouvre la voie au « halal food».

- Dans le cas de la viande bouchère halal, les organisations religieuses islamiques en France n'ont joué jusqu'ici aucun rôle significatif dans la définition des standards de production de viande et produits halal. Ils restent globalement à l'écart du contrôle de la production.

- Le marché de la viande bouchère halal s'est développé en raison de conditions économiques et politiques favorables dans la décennie 90 et été pris en main par les acteurs économiques au dépend des acteurs religieux.

- Le troisième argument est d'ordre démographique sociologique et culturel. Le changement de structure démographique de la population musulmane, sa féminisation notamment ont affecté les conduites d'achat, les pratiques culinaires, et le statut particulier de la viande et celui du sacrifice animal. Ceci a notamment abouti à une minoration de l'acte d'égorgement qui n'est plus un facteur qualitatif important au profit des qualités intrinsèques du produit fini destiné au consommateur.

- En particulier pour les plus jeunes générations, le halal est fréquemment interprété non comme le résultat d'un « processus » d'égorgement, mais comme un « état » de pureté. Le résultat est une configuration favorable pour des initiatives de marketing répondant à des préférences différenciées et évolutives des consommateurs, bref à la naissance en France d'un marché du « halal food ».

\section{Absence de controle religieux sur la fabrication de viande bouchere halal}

5 L'hypothèse selon laquelle les organisations religieuses n'ont, de fait, pas de contrôle sur l'abattage des animaux destinés à la production de viande halal repose sur nos observations des activités d'approvisionnement, d'abattage, et de distribution halal dans les abattoirs et auprès de la totalité des boucheries halal de trois zones de chalandises halal d'Aquitaine, Bordeaux Saint Michel, Agen, Pau (Bergeaud-Blackler, 2001). En raison de directives françaises et européennes, l'abattage d'animaux de boucherie ne peut se faire ailleurs que dans des établissements contrôlés par l'état ${ }^{7}$. Comme il n'existe en Aquitaine aucun abattoir exclusivement réservé à la fabrication de produits casher ou halal, ceux-ci sont produits sur des chaînes d'abattages ordinaires. La différence entre production de viande halal et production de viande ordinaire (non halal et non casher) réside essentiellement dans les outils et instruments utilisés plutôt que dans les étapes de l'abattage qui sont les mêmes. Dans les deux cas l'animal est tué par égorgement et retrait de son sang. Dans le cas du halal, l'animal est égorgé par un opérateur musulman, habilité à sacrifier par une des trois mosquées agréées ${ }^{8}$. Cet opérateur, qui reçoit de la réglementation française, mais pas de la religion musulmane, le titre de « sacrificateur » en raison de son habilitation par l'une des mosquées n'a aucune qualification ou formation particulière ${ }^{9}$. Dans le cas du halal, conformément à la réglementation relative à l'abattage dit rituel, une dérogation à l'étourdissement préalable à la saignée de l'animal peut être utilisée, mais elle n'a aucun caractère obligatoire. En 2000, dans les abattoirs aquitains, la dérogation était systématiquement utilisée pour les bovins. En revanche dans certains abattoirs, les ovins étaient étourdis par électronarcose comme sur la chaîne ordinaire (non halal, 
non casher), la seule différence entre chaîne halal et chaîne ordinaire résidant dans l'identité de l'abatteur (un "musulman» au poste d'égorgement). Pour les bovins l'égorgement était réalisé sur l'animal situé dans un dispositif de contention (casting pen), - obligatoire lorsque la dérogation à l'étourdissement est utilisée - conçu principalement pour protéger l'abatteur des mouvements dangereux de l'animal. La différence entre procédé halal et non halal s'arrête là. Que le sacrificateur choisisse d'abattre avec ou sans étourdissement est une question de convention entre l'abattoir, le chevillard et le sacrificateur mais n'est pas une prescription réglementaire.

6 Nous sommes dans une configuration très éloignée en pratique du cas israélite où des observations et interventions spécifiques sur l'animal sont nécessaires pour qu'il soit déclaré consommables et tamponné « casher » (Nizard-Benchimol, 1997). Dans le cas du casher, les rejets occasionnés imposent un système de traçabilité fiable sur la chaîne. Cela n'est pas le cas sur la chaîne halal qui ne produit aucun rejet. À la fin de la chaîne d'abattage en mode halal, la carcasse est pesée et tamponnée par deux cachets: le tampon légal obligatoire pour toute viande abattue en abattoir, et le tampon halal. Ce dernier est apposé soit par le sacrificateur lui-même, soit par le peseur assermenté de l'abattoir. À ce stade des erreurs de marquage, intentionnelles ou non, peuvent toujours se produire. Les carcasses marquées halal sont ensuite transportées dans des chambres froides pour ressuage ${ }^{10}$, où elles ne sont pas toujours conservées à part des carcasses non halal. Pour les opérateurs présents sur la chaîne d'abattage, les contraintes de production, et les faibles effectifs de main-d'œuvre expliquent le manque de fiabilité du système de traçabilité dans les abattoirs. L'étape suivante de transport est particulièrement propice aux mélanges entre halal et non halal. L'introduction d'abats de carcasses non halal dans des sacs destinés aux boucheries halal par des opérateurs du transport n'est pas rare, comme nous l'avons observé. L'étape suivante est l'arrivée des carcasses dans le frigidaire du boucher. C'est lorsqu'elle franchit le seuil de la boucherie «halal» que la carcasse acquiert définitivement son caractère licite. Avant cela, le statut de la viande reste relativement incertain en raison de la mobilité et les contacts des carcasses avec des espaces non musulmans.

7 Une autre raison, économique celle-ci explique cette incertitude. Les chaînes d'abattage en mode halal produisent souvent une quantité plus importante de carcasses que celle que les détaillants en viande halal ne peuvent en absorber. D'importantes quantités de carcasses dont donc écoulées dans les circuits de viande ordinaire. Il importe dans ce cas de minimiser la visibilité des signes halal. Passé le seuil d'entrée de la boucherie halal, la carcasse acquiert son statut définitif de viande halal qu'elle soit ou non marquée. Car à cette étape, le marquage halal de la carcasse constitue souvent plus une confirmation qu'une preuve. S'il n'y a pas de tampon, l'interprétation du boucher est en général que ce marquage aura pu être mal fait, être effacé, ou avoir été apposé sur un autre morceau de la même carcasse, ou même avoir été oublié compte tenu des cadences industrielles. Les abats en particulier n'arrivent pas toujours marqués ou bagués. Quand bien même le boucher émettrait des doutes sur la licéité de la viande, le coût d'un conflit avec le chevillard ou le livreur, celui d'un renvoi éventuel des carcasses, serait trop élevé pour lui. Dans de nombreux cas le boucher musulman doit sa survie à la confiance passive qu'il a dans l'amont d'une chaîne dont il dépend totalement. 
8 L'observation de l'abattage et du cheminement des carcasses permet d'identifier le seuil à partir duquel une viande devient licite. La viande devient définitivement halal dans la boucherie, un espace qualifié lui-même de halal ou d'islamique. Avant cela son statut est incertain.

\section{Développement en France des marchés halal : conditions économiques, réglementaires et politiques}

9 En 1990, dans une étude réalisée pour le Ministère de l'agriculture, des économistes chiffraient à 200000 et 350000 tonnes de viandes la quantité de viande halal nécessaire à satisfaire la consommation annuelle de quatre millions de Musulmans vivant en France $^{11}$. Notre enquête estimait qu'entre 1996 et 2000, pour le seul quartier Saint Michel de Bordeaux, le nombre de détaillants en viande halal avait doublé1 ${ }^{12}$. Une combinaison de raisons économiques, religieuses et politiques a conduit à la fin des années quatre-vingt-dix à la consolidation des marchés halal quasi inexistants au début des années soixante-dix. Les boucheries halal non seulement se sont multipliées mais elles l'ont fait en partie aux dépens des boucheries traditionnelles et sont à présent durablement inscrites dans le paysage économique urbain.

10 La sévérité accrue des réglementations relatives à l'abattage dans le cadre de l'intégration européenne, l'harmonisation des procédés de production, la recherche de nouveaux débouchés, la souplesse des interprétations relatives à l'abattage rituel musulman ainsi que le retrait des religieux dans la lutte pour le contrôle de la viande halal ont créé l'offre de viande halal.

\section{Rationalisation de la production de viande et quête de nouveaux débouchés}

11 Un des facteurs décisifs de la croissance du marché de la viande halal réside dans la volonté des opérateurs des filières viandes bovines et ovines de trouver de nouveaux débouchés à une marchandise que l'évolution du marché des produits carnés a détournée de son affectation traditionnelle.

12 L'amélioration de la productivité, ainsi que les politiques de "qualité » des produits carnés ont entrainé vers des logiques de filières protégées et donc la production d'animaux non certifiés. Aux animaux de réformes ${ }^{13}$ viennent donc désormais s'ajouter tous les déclassés qui, en raison de leur conformation, âge ou sexe ne peuvent plus être certifiés. Les ventes aux collectivités publiques comme les cantines, hôpitaux et prisons, ou à l'industrie alimentaire du prêt à manger pour fabrication de plats en conserve ou surgelés ne suffisent pas à absorber ces « inclassés ».

13 À cela se sont ajoutés les effets conjoncturels des crises alimentaires sur la demande, effets qui ont touché particulièrement le secteur des produits carnés. La crise de la vache folle a en particulier affecté les exportations de jeunes bovins qui se sont retrouvés en surnombre sur le marché intérieur. Les boucheries islamiques ont ainsi pu acquérir de la viande de jeune bovin à des prix très peu élevés. 


\section{Des réglementations favorables au développement des marchés halal} animaux de boucherie ainsi que des agréments réglementaires de certaines mosquées encadrent depuis 1990 l'abattage rituel musulman. L'ensemble prévoit que tout abattage doit être réalisé par un sacrificateur habilité par un organisme agréé à l'intérieur d'un abattoir équipé de matériel de contention aux normes européennes. Il n'existe aucune réglementation spécifique sur les produits qui en sont issus, notamment en matière de traçabilité et d'étiquetage. Mais toutes les autres réglementations applicables aux produits alimentaires s'appliquent sans aucune exception aux produits halal. Sur le plan des contrôles d'hygiène et de sécurité lorsqu'ils sont effectués - les actions sont beaucoup plus floues ${ }^{14}$. Les pouvoirs publics considèrent qu'il est moins problématique, du point de vue sanitaire et fiscal, que la viande soit abattue en abattoir et vendue en boutique plutôt qu'abattue et distribuée illégalement. années quatre-vingt-dix, constatant le développement rapide du commerce de viande halal, il envisage des solutions de régulation de ce marché pour que les éventuels subsides que pourraient rapporter son commerce ne tombent pas dans les mains de courants islamiques qui ne joueraient pas le jeu de l'intégration républicaine. Le modèle envisagé est celui de la Casherout juive. En 1995, quelques années après les attentats parisiens de septembre 1986, l'affaire Rushdie, la première affaire des foulards islamique de Creil (1989) ${ }^{15}$ et au moment où éclate la guerre civile en Algérie, Charles Pasqua le Ministre de l'Intérieur chargé des cultes, tente de mettre en place un monopole national de la certification halal. Il organise avec le Ministère de l'Agriculture une rencontre entre la Fédération Nationale des Exploitants d'Abattoirs Prestataires de Service (FNEAPS), une puissante organisation représentant les intérêts de l'industrie d'abattage, avec le recteur de la mosquée de Paris, la plus ancienne institution islamique en France. Cette initiative débouchera sur la signature d'une convention de certification halal mais qui ne sera jamais appliquée ${ }^{16}$. Cependant, elle agira comme un signal positif sur les acteurs économiques du secteur des viandes bovines et ovines lesquels parieront alors sur l'existence d'un marché halal national prometteur et lucratif. Nombreux sont les abattoirs qui investissent dans de coûteux box de contention pour abattage rituel et dans l'emploi de sacrificateurs habilités. Le plan Pasqua aura permis également la production de deux arrêtés ministériels. Le premier daté du 15/12/1994 désigne la Mosquée de Paris comme unique organisme religieux agréé pour habiliter des sacrificateurs rituels ${ }^{17}$. Le second date du 27/06/1996 et élargit ce privilège offert par l'État à la mosquée historique, à la Grande mosquée de Lyon et à la Mosquée d'Évry. Ces deux arrêtés ministériels n'auront pas de réelle incidence sur la bataille pour le monopole du marché de la viande halal, en revanche ils marquent le début de la bataille pour le contrôle du marché de la certification halal ${ }^{18}$.

\section{La spirale déflationniste}

La distribution des pouvoirs tout au long de la chaîne se fait au profit de l'amont (producteur et grossiste) et aux dépens de l'aval (détaillants). Vers la fin des années

Revue européenne des migrations internationales, vol. 21 - n³| 2005 
soixante-dix une main-d'œuvre disponible assez peu exigeante (chômeurs maghrébins, anciens ouvriers du bâtiment, des industries métallurgiques ou de l'agriculture) se lance dans le commerce de détail. Ils ouvrent des épiceries comportant des comptoirs de boucherie avec relativement peu d'apport financier initial, grâce au soutien de leurs familles et de leurs réseaux de connaissances. Grâce à des exonérations de charges proposées par l'État ${ }^{19}$, au travail gratuit effectué par les membres de leurs familles, les aides sociales ou encore quelques ventes directes non déclarées, certains parviennent à maintenir voire à développer leur commerce. D'autres subissant une trop forte concurrence, ferment boutique dans l'année qui suit son ouverture. Cette situation de pression ne favorise pas l'entente entre commerçants, et entraîne l'échec de toute tentative de régulation des prix.

Les négociants en viande spéculent sur cette mésentente. Le marché halal étant considéré par la profession du négoce (non musulmane) comme particulièrement incertain et imprévisible, il est traité avec un mépris affiché, et ses potentialités inconnues sont volontairement minimisées. D'après un chevillard bordelais non musulman interrogé, une certaine «éthique » de la profession impose "que l'on ne se dispute pas les "miettes" $\gg 2$. Lorsque, comme c'est souvent le cas en zone rurale comme dans la région d'Agen par exemple, le marché est de dimension réduite le négoce halal est donc tacitement laissé à un seul opérateur lequel, en situation de monopole, a tout loisir de faire pression sur les détaillants qui doivent alors accepter des marchandises à un prix (pour une qualité variable) qui pour la plupart leur permet seulement de se maintenir. La configuration particulièrement défavorable décrite ici se retrouve dans les zones économiques ou les secteurs de la viande et de l'abattage sont en difficulté, et où les populations musulmanes proviennent d'une immigration récente. Dans de grands centres urbains d'immigration ancienne comme à Marseille, où de surcroît la production et la distribution sont dominées par des acteurs musulmans, la situation est bien différente en terme de rapport qualité/prix. L'amont de la chaîne ne domine pas l'aval et les consommateurs peuvent, par l'intermédiaire des détaillants, faire remonter leurs préférences, certes en en acceptant des coûts plus élevés. Il existe entre ces deux extrêmes de nombreuses situations intermédiaires reflétant la diversité structurelle des marchés halal en France ${ }^{21}$.

\section{Désinvestissement des religieux}

Durant les années quatre-vingt, alors que les besoins en infrastructures religieuses (mosquées, écoles coraniques, carrés musulmans dans les cimetières) commencent à s'exprimer publiquement, l'État refuse toute contribution publique au culte musulman arguant de son respect pour la laïcité. C'est là encore le modèle de la Casherout qui va inspirer les responsables d'associations musulmanes dans leur tentative d'instaurer un prélèvement direct sur la viande halal pour financer la création et le maintien des institutions nécessaires à la vie religieuse des Musulmans de France (Bergeaud, 1999). Mais le développement des circuits d'approvisionnement des épiceries-boucheries islamiques est déjà bien avancé. Les commerçants refusent d'entrer dans des querelles « religieuses » dont ils se méfient.

Les années quatre-vingt-dix connaissent un accroissement du nombre des mobilisations dans l'espace public au nom de l'Islam. Des associations de quartier, des groupes piétistes itinérants et des organisations militantes islamiques sont à l'origine 
de ces mobilisations qui recherchent le soutien des communautés émigrées pour faire pression sur les régimes en place de l'autre côté de la Méditerranée (Kepel, 1991; Césari, 1997). Les commerçants, prudents évitent les alliances exclusives avec des mosquées ou associations pour ne pas perdre une clientèle déjà très volatile. Les associations consulaires sont toujours attentives aux affaires économiques de leurs ressortissants mais elles n'ont aucun moyen de pression sur la filière viande française. Les associations socio-culturelles se désintéressent de ce commerce religieux. Quant aux mosquées et organisations explicitement religieuses, elles sont impuissantes tant que la nécessité religieuse de ce commerce n'est pas alors clairement établie. Certaines toutefois favorisent la création d'associations indépendantes de certification halal mais généralement elles n'établissent aucun lien direct avec elles ${ }^{22}$.

\section{« Fausse » viande halal et dynamique de différentiation qualitative}

20 En Aquitaine, dès le début de la deuxième moitié des années quatre-vingt-dix, on assiste à une période de stagnation voire de déflation des prix de la viande halal. Au phénomène de forte concurrence s'ajoute alors une dynamique du soupçon pesant sur la «fausse viande halal». Des boucheries halal ouvrent à un rythme croissant, garantissant toujours être plus «halal » que leurs concurrentes. Le marché arrive à saturation quantitative, les prix sont au plus bas et les crises alimentaires successives ${ }^{23}$ commencent à produire leurs effets sur les pratiques d'achat.

Une différenciation qualitative s'opère alors et les prix commencent à se stabiliser. Le marché entre dans une nouvelle phase. Pressés par leurs clients musulmans mais aussi pour une part non négligeable de non-musulmans ${ }^{24}$, les bouchers ou revendeurs pénètrent peu à peu l'amont. Ils tentent avec plus ou moins de succès de négocier directement sur les marchés ou dans les abattoirs avec les chevillards, imposent aux abattoirs leurs propres employés au poste de sacrificateur (en dépit des réglementations qui imposent que ces sacrificateurs soient habilités par des mosquées agréées) et, mieux au fait des techniques locales de découpe, exigent des carcasses à meilleur rendement. À l'intention de leurs clients, ils décorent leurs boutiques avec des placards publicitaires conçus pour la boucherie traditionnelle locale, et contre le bœuf britannique suspecté d'ESB, signalent sur la devanture à côté du signe halal désormais plus visible, que leur viande est « cent pour cent » française. Dans le Sud-ouest, alors que le boucher halal subit une situation de compétition intense qui l'empêche de contrôler les prix et la qualité des carcasses, l'acheteur bénéficie, lui, d'une situation un peu plus avantageuse. Les différenciations qualitatives, même timides, lui offrent un éventail de choix plus élargi pour des prix qui globalement n'augmentent guère. Sur les marchés, les plaintes et rumeurs de fausse viande halal se développent avec pour vertu de dynamiser encore un peu plus le commerce, en poussant les commerçants à des efforts sur les prix et le service. Mais ces efforts sont limités lorsqu'il est question de qualité. La viande halal quand elle n'est pas une marchandise de dégagement, reste de qualité variable, passant du meilleur au pire sans claire logique du point de vue du consommateur. L'incertitude qualitative pèse négativement sur le niveau de confiance. 


\section{Minoration de l'acte d'égorgement}

Il existe une certaine autonomie entre les conditions de production effectives de viande halal et le discours des mangeurs. Les méthodes de production de viande halal sont peu connues des acheteurs, ce qui ne les empêche pas d'en parler. Ils sont comme tous consommateurs de viande peu empressés de savoir ce qui se passe dans un abattoir (Vialles, 1987), mais en même temps c'est en référence à un mode spécifique d'abattage qu'ils justifient leur conduite d'achat et de consommation. "La viande halal est égorgée » affirment les acheteurs sur le marché Saint Michel de Bordeaux ${ }^{25}$, comme si la viande non halal ne l'était pas $^{26}$. Pour la viande bovine, la preuve de la licéité résiderait notamment dans la clarté de la viande. étant égorgée, la bête serait vidée de son sang et sa viande serait claire. Une viande non halal aurait a contrario conservé son sang ce qui lui donnerait une couleur foncée. En réalité cette différence de couleur est attribuable au type de bête fourni aux bouchers halal ainsi qu'à la différence de durée de maturation de la viande en boucherie. Les bouchers musulmans s'approvisionnent de préférence en viande de jeunes bovins, viande rosée qui se vend mal dans les boucheries non musulmanes. Trop claire, ni blanche, ni rouge, elle est boudée par l'acheteur traditionnel bordelais non musulman car elle n'est à ses yeux « ni veau ni bœuf $»^{27}$. De plus la viande bovine de boucherie halal est vendue de préférence très fraîche, alors que celle vendue dans la boucherie ordinaire aura maturé pendant au moins une semaine ce qui lui donnera une couleur plus foncée (Bergeaud, 2000).

Les changements sociologiques intervenus dans la population musulmane au cours des trente dernières années de naissance et de développement de ce marché communautaire expliquent en partie cette autonomisation relativement rapide des marchés de viande halal. D'abord, les femmes émigrées, "les cuisinières ", venues rejoindre leurs époux par la procédure de regroupement familial, ont joué un rôle décisif dans la formation des commerces maghrébins en émigration, et dans celle de l'offre des produits. La viande a changé de statut dans la cuisine maghrébine confrontée à d'autres traditions culinaires. Ensuite, les hommes maghrébins sont exclus des processus de production tenus par des entreprises extracommunautaires. La cuisine et la distribution ont désormais la primeur sur la production, les cuisinières sur ceux qui n'ont plus le monopole de la production.

\section{La viande des cuisinières}

En deux décennies, depuis leur arrivée à la fin des années soixante-dix, la cuisine des femmes maghrébines ${ }^{28}$ a sauvé de la disparition une partie du commerce de centreville, qui disparaissait peu à peu au profit de la grande distribution. Le cas du quartier Saint Michel de Bordeaux est exemplaire (Bergeaud-Blackler, 2000). Des réseaux d'approvisionnement et des points de distribution se mettent en place pour fournir à ces cuisinières les produits nécessaires à la réalisation de leurs recettes. Les produits vivriers (fruits et légumes, viandes et produits animaux) proviennent de la région, les épices, fruits secs, thés, farines, boîtes de conserve et certaines céréales de circuits d'approvisionnement plus larges, profitant à toutes les communautés africaines, turques ou asiatiques de France.

L'essentiel des denrées dont la cuisine des Maghrébines a besoin est disponible sans qu'il y ait besoin de construire des canaux d'importation spécifique depuis les pays 
d'origine. Grâce aux informations sur les qualités des produits qui circulent de « bouche à oreille ", les femmes, guère informées par un étiquetage français, chinois, turc ou même arabe, apprennent à distinguer et choisir les produits qui flatteront le mieux leur cuisine. L'offre de produits alimentaires se constitue ainsi progressivement à partir de la sélection des cuisinières et les vendeurs s'efforcent comme ils le font au Maghreb d'être réactifs à leur demande. Dans les épiceries maghrébines en France comme de l'autre côté de la Méditerranée, le bon vendeur se reconnaît à sa capacité de, dénicher parmi les innombrables boites rangées les unes sur les autres du sol au plafond, le produit justement désiré. Pour les viandes destinées aux tagines et au couscous, les cuisinières ont une préférence pour les morceaux de viande très fraîche laissant paraître une chair rosée, et molle rappelant l'état pantelant de la carcasse d'un animal juste abattu, très éloignée de celle bien tenue, foncée, terne et « rassise ${ }^{29}$ dans lesquels sont découpés de formes régulières, présentées ensuite en rangées dans les vitrines des boucheries de tradition française (Bergeaud-Blackler, 2000). Pour que cette viande non maturée soit tendre et pas trop longue à cuire, il est préférable qu'elle provienne d'un animal jeune. Le choix d'avants de carcasse de jeunes bovins facilite également le travail des épiciers-bouchers halal qui maîtrisent mal l'art de la découpe et sont peu experts de la nomenclature bouchère ${ }^{30}$. Quand les morceaux découpés n'ont pas été achetés dans les trois jours après livraison et qu'ils commencent à prendre une teinte foncée, la viande est hachée pour être écoulée rapidement en kefta et merguez avant le nouvel arrivage de carcasses fraîches.

Les bacs où trempent les olives, ainsi que les sacs d'épices sont placés à proximité des vitrines réfrigérantes, c'est-à-dire dans l'espace des produits frais, pour montrer leur qualité de fraîcheur. En retour, la viande bénéficie du «flavor principle » (Rozin and Rozin) ${ }^{31}$, c'est-à-dire ce complexe olfacto-gustatif spécifique de la cuisine du Maghreb, donnant à celui qui pénètre dans l'échoppe le sentiment que la viande, elle aussi, pourrait provenir du pays d'origine. Le travail de maîtrise et d'apprêt de la matière carnée dans laquelle subsiste peu d'os procure une forte impression aux clientes. Ces amas de chair de viande fraîche donnent une impression de richesse, de profusion et de satiété.

\section{La viande, un aliment ordinaire}

27 Le prix de la viande est en France moins élevé relativement au salaire que dans les pays du Maghreb où elle ne constitue un produit de consommation régulière que pour une minorité de familles citadines ${ }^{32}$. En France, qu'elles habitent en ville ou à la campagne, les familles immigrées peuvent généralement acheter de la viande et pour certaines en consommer de façon régulière voire à tous les repas. Si certaines variations de prix existent entre boucheries, en particulier depuis la période récente de différenciation qualitative, elles ne reflètent pas de segmentation du marché comme c'est le cas au Maroc par exemple où les différents types de boucheries s'adressent à différents groupes sociaux (Bergeaud-Blackler, 2002).

28 Au Maghreb, la différenciation chair d'un côté et d'os et gras de l'autre fonde la distinction entre d'une part la cuisine du pauvre - où les trois composantes : os, gras et chair sont utilisés en petite quantité comme condiment et exhausteur de goût, et d'autre part la cuisine du riche (ou de l'événement d'exception) où la quantité de chair domine sur l'os et le gras. Cette distinction n'existe plus en émigration. La 
consommation de chair de viande n'est plus exceptionnelle et cela n'en fait plus un marqueur social.

\section{L'Aïd el Kebir : la fête de la viande}

Pratiqué en dehors d'un abattoir, l'acte d'égorgement de l'animal est devenu en émigration un fait illégal, clandestin ${ }^{33}$, alors qu'il était traditionnellement un acte d'honneur masculin. En France, s'il est encore pratiqué par les hommes vivant en milieu rural - et par une minorité de familles citadines - il a obligation d'invisibilité tout comme le commerce qu'il peut engendrer. L'acte de mise à mort et celui de la distribution traditionnellement dévolue à l'homme tendent à disparaître y compris lors de la fête de l'Aïd el Adha, fête du sacrifice (Bonte, 1999). Les études menées en France et en Europe au cours des vingt dernières années (Brisebarre, 1998) attestent de la très grande popularité de cette pratique parmi les familles musulmanes maghrébines et africaines sub-sahariennes. Mais si les étapes du rituel (quête de l'animal, préparation de l'animal, sacrifice, partage, cuisine rituelle) sont réadaptées aux nouveaux contextes par les familles, les sacrifiants, c'est-à-dire ceux pour le compte de qui le sacrifice est effectué, sont de plus en plus nombreux aux dépens des sacrificateurs. Autrement dit la délégation du sacrifice tend à devenir la règle plus que l'exception. La réglementation dessaisit le chef de famille de sa prérogative de « sacrificateur » en obligeant le sacrifice à être délégué à un sacrificateur habilité et à être effectué en abattoir. Les associations islamiques de leur côté insistent sur la manifestation d'obéissance à Dieu que représente l'acte rituel et sans aller jusqu'à déconseiller sa pratique "hors la loi ", offrent des alternatives à sa réalisation comme par exemple un don monétaire pour le sacrifice d'un mouton en Tchétchénie ou en Irak dont la viande pourrait nourrir là-bas une famille musulmane. La cuisine rituelle de l'Aïd el Kebir (la grande "fête»), dimension commensale, collective et féminine, prend le pas sur le sacrifice rituel de l'Aïd el Adha (la fête du "sacrifice»), dimension religieuse, individuelle et masculine. Le sacrifice s'oriente vers la commensalité (Detienne, 1979). L'amont du sacrifice s'estompe pour donner de l'importance à la matérialité de l'animal. C'est l'Animal qui est désormais au centre de la fête ${ }^{34}$ ou de ses controverses.

En conclusion, l'importance que le développement de la cuisine maghrébine et le pouvoir des cuisinières ont attribuée à la matière carnée dans le commerce halal, l'indifférenciation entre méthode d'abattage halal et non halal ainsi qu'une conception de plus en plus zoocentrée du sacrifice entraînent vers une représentation particulière de la viande «halal ». Halal devient une chose, et non plus un attribut, une matière pure. L'égorgement n'est plus le processus central de transformation.

On assiste à une réactivation du tabou du porc et de l'interdit de l'alcool, ainsi que de leurs dérivés ou «contaminés ». On passe ainsi progressivement de la viande égorgée au nom d'un Dieu unique, au marché des aliments religieux : le halal food.

\section{Dynamique de différenciation et nouvelle conception du halal}

Une des raisons de la dynamique rapide de création des boucheries islamiques est l'absence de viande de porc. Si les doutes subsistent sur la façon d'égorger l'animal, l'acheteur sait en revanche qu'en se rendant dans une boucherie islamique il ne 
rencontrera aucun produit d'origine porcine. Les jeunes générations, peut-être parce qu'elles sont volontiers plus souvent consommatrices de plats cuisinés, ou parce qu'elles sont tributaires de la cuisine d'autrui, se montrent particulièrement concernées par la présence de porc ou de ses contaminés dans les aliments qu'elles achètent. Cette attention à l'égard du contenu des aliments s'inscrit dans une tendance plus générale exprimée par d'autres groupes de consommateurs comme les végétariens, les consommateurs de produits biologiques, macrobiotiques, ou crus. Le halal est un principe de discrimination certes exclusif mais il est loin d'être réducteur puisqu'il ouvre aux Musulmans de toutes générations et de tous pays, toutes les cuisines du monde. Les nouveaux produits «halal» de l'Islam "mondialisé » (Roy, 2002) peuvent aujourd'hui circuler d'autant plus facilement à travers les cinq continents que le «halal» est encadré par l'instance de référence de l'OMC: le codex alimentarius, essentiellement pour prévenir toute barrière aux échanges.

\section{Le tabou du porc}

Le tabou du porc est un tabou antéislamique déjà connu par le Judaïsme et d'autres cultes généralement situés dans la zone du Proche et Moyen Orient (Simoons, 1994). Inscrit dans le texte coranique de façon tout à fait explicite ${ }^{35} \mathrm{il} \mathrm{a} \mathrm{toujours} \mathrm{été} \mathrm{largement}$ respecté par la majorité des Musulmans (Simoons, 1994:35-36). Quand cet interdit a été transgressé, il s'agissait le plus fréquemment d'une pratique de contestation de l'autorité religieuse. Pour l'anthropologue Mohammed Benkheira (2000), la prohibition du porc est si bien intériorisée chez les Musulmans maghrébins qu'il faut la distinguer qualitativement de celle s'appliquant à l'alcool. Alors que cette dernière est explicite, proclamée, consciente, volontaire et débouche sur une répression, l'interdit de consommation de la viande de porc est intériorisé, digéré, comparable dans son intensité à la prohibition de l'inceste : «nous n'évitons pas l'inceste seulement parce que les lois, écrites ou non, l'interdisent et donc parce que nous craignons un châtiment si nous violons la loi, mais surtout parce que l'idée même de l'inceste nous répugne et nous révolte » (Benkheira, 1995 : 67-88). Autrement dit, le dégoût du porc dans les sociétés musulmanes serait si profond qu'il ne serait même pas utile d'en interdire la consommation.

Les périodes récentes de crises alimentaires ont réactivé le tabou du porc dans les discours des Maghrébins vivant en France. Comme dans le reste de la population générale des consommateurs, on voit s'exprimer une étiologie spontanée de ces «nouvelles maladies » de l'animal, avec pour cette population musulmane une mise en parallèle avec le tabou islamique du porc. Pour certaines personnes interrogées lors de la fête de l'Aïd el Kebir en 2000, la fièvre aphteuse était considérée comme une maladie d'origine porcine. En pleine crise de l'épizootie de fièvre aphteuse un tiers des habitués de l'abattage direct ont renoncé au sacrifice ou bien se sont rendus à l'abattoir, pour s'approvisionner en bêtes certifiées par la Direction des Services Vétérinaires (Bergeaud-Blackler, rapport, 2001).

\section{Le halal devient peu à peu synonyme de pureté}

Le contenu des aliments est désormais scruté. La recherche de dérivés de porc et d'alcool est devenue un nouveau sujet de discussion. Les forums de discussion musulmans sur Internet véhiculent, développent et en même temps « démocratisent » 
le débat sur la frontière entre mangeable et immangeable, licite et illicite du point de vue islamique. Ces sites sont un lieu d'observation intéressant sur l'islam vécu des jeunes $^{36}$. Nos investigations systématiques des messages envoyés aux forums de discussion des deux sites internet ${ }^{37}$ montrent que les aspects techniques de l'abattage sont moins souvent objet de questionnements que la composition des aliments d'une part (Cet additif est-il permis? Tel produit contient-il du porc?) ainsi que le fait de savoir où placer les frontières de la convivialité (puis-je manger la cuisine de ma belle-sœur qui n'est pas musulmane? mes beaux-parents mangent du porc, comment les convaincre que c'est haram ?). La possibilité d'interprétation directe des textes religieux par les croyants eux-mêmes établit un rapport direct et individuel à la religion, et renforce le sentiment d'autonomie et de liberté religieuse. Mais lorsque ces interprétations sont multiples et contradictoires, elles peuvent aussi constituer un univers menaçant, particulièrement lorsque cela touche des questions aussi fondamentales que la commensalité. La pluralité des interprétations, et les nouveaux canaux de diffusion que sont les sites internet alimentent des débats anxieux. Par exemple, pour certains l'extrait coranique "la nourriture des gens du Livre (chrétiens et juifs) vous est permise» autorise le partage du repas avec des non musulmans à condition de s'abstenir de manger du porc. Une autre lecture du même verset permet le partage d'un repas avec un non musulman mais le subordonne à l'assurance que ce qui est offert ne contient pas de porc ou ses dérivés. Une autre lecture oppose à cette dernière interprétation le principe de l'abstention dans le doute, celui de la souplesse de l'Islam dont Dieu a «facilité » la pratique et prétend que l'on ne doit pas soupçonner et donc s'abstenir de demander la composition de ce qu'on mange. "Chercher la connaissance jusqu'en Chine » est une injonction religieuse répétée à l'envie par les internautes musulmans. Au lieu de rassurer, cette quête devient anxiogène, en particulier quand il est question de pratiques aussi quotidiennes et intimes que l'alimentation. À la fin, une certitude domine qui tranche sur les nuances évoquées. Tout le monde semble la partager: le porc et l'alcool sont haram ${ }^{38}$. La cyber-surveillance sur le contenu, la pureté des aliments, s'exerce à l'échelon planétaire. Des sites sont créés uniquement pour dénoncer les «gabegies » et les tromperies concernant le commerce halal.

\section{Naissance du halal food}

La diversification croissante de la clientèle des bouchers halal et la forte intégration des circuits de production et distribution de la viande qu'ils commercialisent à la filière viande ordinaire n'ont pas motivé jusqu'ici ces commerçants à entreprendre une démarche collective de qualification. En position défavorable par rapport à l'amont de la chaîne de production, les bouchers ne sont guère en mesure de s'entendre pour créer un label halal unique. La mise en place d'un tel certificat renchérirait les prix au détail qui pourrait conduire à la fermeture de nombreux points de vente. Tant que les supermarchés ne jouent qu'un rôle réduit dans le secteur de la distribution halal, la situation telle qu'elle s'est développée est pour eux garante d'un équilibre certes précaire mais grâce auquel ils peuvent se maintenir.

37 Jusqu'au tournant de l'année 2000, sur la plus grande partie du territoire français, la viande de boucherie se vendait dans le cadre de conventions «domestiques» (MAD, 1995) et le boucher était le principal garant de la fraîcheur de la viande autant que de la licéité du procédé d'égorgement. Mais cette situation a changé récemment avec le développement national et international du marché de la certification halal. Ce sont 
d'abord les opérateurs de la filière de production des volailles ${ }^{39}$ dont l'objectif est d'abord la vente en supermarché qui se sont lancés dans la certification industrielle en proposant leurs propres labels halal. Les différents maillons de la chaîne de production, liés par des «conventions industrielles ${ }^{40}$ » écrites anonymes, ne se substituent à aucune fonction traditionnelle. Il n'existe pas de « boucher » de la volaille, la mise à mort étant à l'origine une partie du processus culinaire et non un préalable à celui-ci ${ }^{41}$.

Aujourd'hui des labels halal existent également pour les viandes bouchères (bovines et ovines) fraîches et congelées et pour les produits carnés. Ils sont conçus et proposées aux industriels de l'agro-alimentaire par les trois mosquées qui ont le monopole de la distribution des cartes de sacrificateurs, mais aussi des nombreuses sociétés de contrôle spécialisées ${ }^{42}$. Ces labels ont d'abord été conçus pour l'exportation vers des pays musulmans. Le commerce international des produits halal s'est développé si intensément au cours des années quatre-vingt-dix que le Codex Alimentarius ${ }^{43}$ a adopté le 17 Juillet 1997 un guide sur l'usage de la mention « halal » afin que les différences de conception n'entravent pas les échanges commerciaux mondiaux ${ }^{44}$. Dans les discours des immigrées de la première génération, l'égorgement est le moment symbolique primordial par lequel une viande acquiert son statut halal, et ce de façon définitive. Le guide du Codex, qui a été conçu à partir de références différentes de celle de l'Islam maghrébin, insiste sur la nécessité pour un produit halal d'être dépourvu de toute substance haram (illicite) et également de n'avoir pas été en contact avec des produits non-halal ${ }^{45}$. Cela signifie que dans le système du " halal food» tel qu'interprété par le Codex, l'aliment halal peut redevenir impur, corrompu par contamination volontaire ou involontaire. Cette conception entraîne vers une logique de filière séparée où la séparation des produits, et plus seulement des viandes, sera aussi nécessaire que la spécificité de l'acte d'égorgement ${ }^{46}$. L'arrivée prochaine de la grande distribution dans ce secteur devrait renforcer cette conception du « worldhalal» food.

\section{Conclusion}

La qualité d'un produit n'est pas définie une fois pour toutes entre le producteur et le consommateur. Elle n'est pas déterminée avant sa production, mais découle plutôt des conditions de celle-ci et donc des conventions entre les différents acteurs de sa production et de sa consommation. Les marchés halal, mais aussi les normes du halal sont progressivement redéfinis par les différents opérateurs de l'agro-alimentaire en fonction des opportunités des marchés régionaux, nationaux et internationaux. Les organisations religieuses prennent une part relativement réduite dans ce commerce ou dans son contrôle et lorsque des voix se lèvent de temps à autre pour pointer l'absence de règles claires, elles sont le plus souvent considérées comme un avatar inévitable des modalités de l'échange commercial. Soit les consommateurs sont considérés comme des dupes, soit on pointe leur complicité face à des pratiques "corrompues " (cf. les nombreux discours sur le "faux» halal qui ne s'appuient sur aucune définition du halal). En démontrant que la qualité d'un produit se construit tout au long de la vie, la théorie des conventions ouvre le champ à d'autres perspectives. Aujourd'hui le rapport de force est en faveur de l'amont sur l'aval. Dans de nombreuses régions de France comme en Aquitaine, la situation tient principalement à la survie des détaillants bouchers épiciers. Mais le changement de conception du halal chez les jeunes générations pourrait s'accompagner d'un renversement des rapports de pouvoirs au 
long de la chaîne de production-distribution en faveur de l'aval et plus spécialement de la grande distribution. Cela pourrait modifier en profondeur l'organisation des circuits de production et conduire à une intégration amont-aval et donc une plus grande spécialisation du halal sur le mode d'une «filière qualité ». L'enjeu réglementaire pour les années à venir pourrait être de parvenir à une distribution plus équitable des pouvoirs le long de la chaîne, passant par l'acceptation du rôle que les consommateurs et les référents religieux seraient en droit de jouer dans la définition de ces produits. Les controverses récentes sur la légitimité de l'abattage rituel musulman dans de nombreux pays européens (Bergeaud-Blackler, 2004b) devraient dans un futur proche entraîner vers une réflexion sur le statut de ces produits. Sont-ils des produits commerciaux, des produits religieux ou les deux? De la réponse dépendra en partie le type de réglementation (privé/publique, product or process driven) à appliquer pour que la transparence et la protection des consommateurs, une des priorités nationales et européennes de cette période d'après crise de la vache folle, soient ici comme ailleurs, mieux prise en compte.

\section{BIBLIOGRAPHIE}

BEARDSWORTH Alan and KEIL Teresa (1997) Sociology on the Menu : An Invitation to the Study of Food and Society, London, Routledge, $277 \mathrm{p}$.

BENKHEIRA Mohammed-Hocine (1995) Lier et séparer. Les fonctions rituelles de la viande dans le monde islamisé, L'Homme, $\mathrm{n}^{\circ}$ 52, pp. 89-114.

BENKHEIRA Mohammed-Hocine (2000) Islam et interdits alimentaires : juguler l'animalité, Paris, PUF.

BERGEAUD Florence (1999) L'institutionnalisation de l'Islam à Bordeaux. Enjeux sociaux, politiques et économiques de l'implantation du culte musulman dans un espace urbain, thèse de doctorat, Université de Bordeaux 2, $501 \mathrm{p}$.

BERGEAUD-BLACKLER Florence (2000) Le goût de la viande halal : viande de boucherie française et viande de boucherie marocaine, Bastidiana. - Brionne, $\mathrm{n}^{\circ}$ 31-32, pp. 147-158.

BERGEAUD-BLACKLER Florence (2001) «La viande halal peut-elle financer le culte musulman?» Le Journal des Anthropologues $\mathrm{n}^{\circ} 84$.

BERGEAUD-BLACKLER Florence (2001) Production et consommation de viande halal en Aquitaine Rapport pour DGAL- Santé et Protection animale, Ministère de l'Agriculture et Aquibev, 450 pp. inc. illustrations.

BERGEAUD-BLACKLER Florence (2002) «Viande b'ldi et viande rumi : l'origine de la découpe de la viande halal en France » Slow, février.

BERGEAUD-BLACKLER Florence (2004) Qui sont les consommateurs de viande halal ? Les Cahiers de l'OCHA (Publication du Cidil) Paris.

BERGEAUD-BLACKLER Florence (2004b). Nouveaux enjeux autour de l'abattage rituel : une perspective européenne, Cahiers d'Economie et de Sociologie Rurales (73). 
BERGEAUD-BLACKLER, Florence (2005) l'Etat, le culte musulman et le halal business, Actes du colloque Droits, libertés et obligations du culte musulman, Journées d'études «Islam et société ", EHESS, Institut d'études de l'Islam et des Sociétés du Monde Musulman, Paris. (À paraître).

BONTE Pierre, BRISEBARRE Anne-Marie et GOKALP Altan (1999) Sacrifices en Islam. Espaces et temps d'un rituel, Paris, CNRS Éditions, 465 p.

BONTE Pierre (1999) Sacrifices en Islam : textes et contextes in Brisebarre Anne-Marie, Gokalp Altan dir. Sacrifices en islam. Espaces et temps d'un rituel, Paris : CNRS Éditions, 465 p.

BUNT Gary R. (2003) Islam in the Digital Age : E-jihad, Online Fatwas and Cyber Islamic Environments, London, Pluto Press.

BRISEBARRE Anne-Marie (1998) La Fête du mouton, un sacrifice musulman dans l'espace urbain, Paris, CNRS Editions.

CAEIRO Alessandro (2003) The European Council for Fatwa and Research, European University Institute - Robert Schuman for Advanced Studies.

CESARI Jocelyne (1994) Etre musulman en France aujourd'hui, Karthala et Iremam, 367 p.

CORBEAU Jean-Pierre (1994) Le manger, lieu de sociabilité. Quelles formes de partage pour quels types d'aliments? Pratiques Alimentaires et Santé, in Prévenir n² 26, pp. 203-218.

DASSETO Felice et BASTENIER Albert (1980) L'Islam transplanté : vie et organisation des minorités musulmanes en Belgique, Anvers, EPO, 203 p.

DETIENNE Marcel (1979) Pratiques culinaires et esprit de sacrifice, in Détienne Marcel, Vernant Jean-Pierre éd. La cuisine du sacrifice en pays grec, Publication du centre de recherches comparées sur les sociétés anciennes, Paris, Gallimard, pp. 7-35.

DOUGLAS Mary (1966) De la souillure : essai sur les notions de pollution et de tabou ; trad. de l'anglais par Anne Guérin, Paris, François Maspéro, 1971.

FABRE-VASSAS Claudine (1994) La Bête singulière. Les Juifs, les chrétiens et le cochon, Paris, Gallimard, coll. Bibliothèque des sciences humaines, $418 \mathrm{p}$.

HAL Fatema (1996) Les Saveurs et les Gestes, Cuisines et Traditions du Maroc, Paris, Stock, 239 p.

International Markets Bureau - Market and Industry Services Branch Agriculture and Agri-Food (2002) Halal Food Products Market Report, Canada official website,

http://atn-riae.agr.ca/info/africa/e3281.htm

JAOUADI Tahar (2000) Evolution du comportement alimentaire tunisien, in Padilla M. Oberti B. éd., Alimentation et Nourritures autour de la Méditerranée, Paris, Karthala et CIHEAM, 264 p.

KALTENBACH Jeanne-Hélène et TRIBALAT Michèle (2002) La République et l'islam, entre crainte et aveuglement, Paris, Gallimard, $340 \mathrm{p}$.

KEPEL Gilles (1991) Les Banlieues de l'Islam, Naissance d'une religion en France, Paris, Seuil, réed. de 1987,425 p.

LEVEAU Rémy dir. (1998) Islam(s) en Europe, Approches d'un nouveau pluralisme culturel européen, Berlin, collection des travaux du centre Marc Bloch.

LEVEAU Rémy, MOHSEN FINAN Khadija et WITHOL DE WENDEN Catherine (2001) L'Islam en France et en Allemagne : identités et citoyennetés, La Documentation française. 
LUPTON Sylvie (2003) Conventions et incertitude partagée sur la qualité des biens. Colloque Conventions et Institutions Approfondissements théoriques et contribution au débat politique, Université Paris X Nanterre. 11-13 Décembre 2003.

MARSHALL Alfred (1920) Principles of Economics, London.

MOUREAU Nathalie SAGOT-DUVAUROUX Dominique (2003) La construction sociale d'un marché : l'exemple du marché des tirages photographiques, Colloque Conventions et Institutions Approfondissements théoriques et contribution au débat politique, Université Paris X Nanterre. 11-13 décembre 2003.

NEFUSSI, Jacques (1996) Le marché de la viande halal en France, Rapport de la Mission Agro Développement (MAD) pour la DGAL Ministère de l'Agriculture, 1996.

NIZARD BENCHIMOL Sophie (1997) L'économie du croire, une anthropologie des pratiques alimentaires juives en modernité, Thèse de doctorat, Ecole des Hautes Etudes en Sciences Sociales 515 p.

OUBROU Tarek (2001) Entretien avec. La Medina, la viande halal, la face cachée, numéro spécial nº 5.

Al-QARADAWI Yousse (1960). The Lawful and The Prohibited in Islam, Tr : El-Helbawy, Siddiqui, Shukry et al., 355 p.

ROY Olivier (2002) L'Islam mondialisé, Paris, Edition du Seuil, 210 p.

ROZIN Paul (1982) Human Food Selection : The Interaction of Biology, Culture and Individual Experience in Barker, Lewis M. eds, The Psychobiology of Human Food Selection. Westport, AVI Publishing Company, pp. 225-254.

SIMOONS Frederick J. (1994) Eat not this flesh, The University of Wisconsin Press, pp. 13-102.

VIALLES Noelie (1987) Le sang et la chair. Les abattoirs des pays de l'Adour, Paris, Maison des Sciences de l'Homme/Mission du Patrimoine Ethnologique, $160 \mathrm{p}$.

\section{Sites internet}

http://www.oumma.com

http://www.islamie.com

http://www.uoif-online.org

\section{NOTES}

1. Les logiques socialisatrices à l'œuvre à travers la scolarisation affectent les façons de communiquer, de se nourrir, se vêtir, d'être ensemble chez les individus quelles que soient leurs origines culturelles et religieuses. Dans le cas des enfants issus de l'immigration maghrébine, ces façons d'être entrent parfois en conflit avec les valeurs familiales musulmanes, ce qui conduit certains à rechercher un arbitrage religieux. Le sociologue A. Caeiro remarque que les musulmans en Europe sont « d'avides consommateurs de fatwas » (Caeiro, 2003).

2. Ainsi sont nommés juifs et chrétiens, adeptes de religions bibliques.

3. Entretien avec M Thami Breze à son domicile à Cenon en mars 1998. C'est également opinion de l'imam Tarek Oubrou interrogé par l'auteure in La Medina (2000).

4. Les estimations rapportées dans la presse sont généralement très imprécises, parfois fantaisistes : 95 \% de la viande halal est non halal affirme The Middle East magazine en juillet 1996 
(repris par Courrier International). Plus tard l'Express se fait l'échos d'un fonctionnaire anonyme du Ministère de l'Intérieur qui affirme que $50 \%$ de la viande vendue (à Rungis) finit dans les boucheries islamiques, Rungis comptant très peu de grossistes en produits carnés halal. (L'Express du 21/11/2002). Ces informations ne sont pas référées et sont donc incontrôlables, ce qui est le propre des rumeurs.

5. "“Une véritable mafia", "Du racket”, "Des consommateurs abusés" : pour une fois, imams, recteurs de mosquée, dirigeants d'associations religieuses, bouchers de quartier, grossistes en viande, tous sont d'accord. L'objet de leur indignation? L'affairisme qui gangrène le marché français de la viande sacrifiée selon le rite islamique, dite halal (licite)». Extrait de «L'argent de l'Islam » par Besma Lahouri et Boris Thiolay dans l'hebdomadaire L'Express du 21/11/2002.

6. Ce point pourrait toutefois être discuté puisque des consommateurs affirment pouvoir distinguer à l'aide de leur sens visuels, gustatifs ou encore olfactifs une viande halal d'une viande non halal (Bergeaud-Blackler, 2000).

7. À quelques exceptions près mais elles ne concernent pas nos propos ici. Cf. «Conditions de l'abattage rituel en France» MHR Viande. http://www.mhr-viandes.com/fr/docu/docu/ d0002083.htm

8. Conformément à l'art. 13 du décret du $1^{\text {er }}$ octobre 1997 : l'abattage rituel ne peut être effectué que par des sacrificateurs habilités par les organismes religieux agréés, sur proposition du ministre de l'intérieur, par le ministre chargé de l'agriculture. (...) Les sacrificateurs musulmans sont habilités par les mosquées qui bénéficient de l'agrément d'organismes religieux habilitant des sacrificateurs rituels. Les organismes concernés sont: la Grande Mosquée de Lyon et la Mosquée d'Évry par arrêté du 27 juin 1996, et la Grande Mosquée de Paris par arrêté du 15 décembre 1994 (modifié par arrêté du 24 novembre 1995).

9. Selon les autorités musulmanes, en principe n'importe quel fidèle de bonne moralité, peut égorger, il n'existe pas de titre ou de fonction spécifique.

10. La réglementation recommande que la durée de ressuage à l'abattoir des carcasses soit d'au minimum de quarante-huit heures à une température déterminée.

11. Étude de la SOFRES et de la MAD pour la DGAL (Ministère de l'Agriculture).

12. Passant de 12 à 24 pour le seul quartier Saint Michel du vieux Bordeaux (Bergeaud, Rapport, 2001).

13. Les animaux de réforme sont ceux qui ne sont plus destinés à ce pour quoi ils ont été produits en priorité. Par exemple, une brebis dite allaitante ne pouvant plus allaiter est envoyée à l'abattoir en tant qu' " animal de réforme».

14. Lorsque nous enquêtions sur les boucheries islamiques à Bordeaux, la Direction des Services Vétérinaires connaissait l'existence de cinq détaillants bouchers halal lorsque nous-mêmes en avions recensé vingt.

15. Dans un collège de Creil, des collégiennes avaient refusé d'ôter leurs foulards pendant les cours sur demande des enseignants et du proviseur. Pour la première fois des associations musulmanes avaient pris ouvertement position pour les soutenir.

16. En définitive, les conditions de la convention étaient beaucoup moins avantageuses pour eux que les contrats qu'ils avaient déjà passé depuis parfois de longues années, à l'échelon local, avec des groupes de musulmans de leurs régions. Voir Bergeaud-Blackler, 2000.

17. Les sacrificateurs ne seront plus titularisés par les préfectures mais par la Mosquée de Paris.

18. Cf. différence entre marché halal et marché de la certification (Bergeaud-Blackler, 2005).

19. Exonérations applicables aux commerces s'installant en zone défavorisée ou applicables la première année à toute entreprise.

20. Interview avec un négociant en viande, réalisée en février 2000 à Bordeaux. Chez les professionnels de la viande le discours sur le halal est presque toujours de dénigrement, de minoration. 
21. Nous avions conclu dans notre rapport sur les marchés de viande halal en Aquitaine que la situation de ces marchés (au pluriel) dépendaient d'un certain nombre de facteurs dont le facteur économique régional, celui historique et démographique de l'immigration (Bergeaud-Blackler, 2001).

22. C'est le cas par exemple de l'organisme de certification AVS soutenue par des organisations comme l'UOIF avec qui elle n'a aucun lien organique.

23. Encéphalopathie Spongiforme Bovine (ou «maladie de la vache folle»), Dioxine, Fièvre aphteuse etc.

24. Dans certains quartiers urbains les boucheries halal ont remplacé les boucheries non halal qui ont fermé au profit des grandes surfaces. On y trouve une clientèle non musulmane.

25. Cf. également les résultats de notre enquête consommateurs sur le marché bordelais (Bergeaud-Blackler, 2004).

26. Dans un abattoir industriel, en mode halal ou non halal, les animaux sont saignés, c'est-à-dire vidés de leur sang.

27. En particulier dans le Sud Ouest où une bonne viande de bœuf doit être de préférence de couleur rouge foncée.

28. La cuisine maghrébine en France ne va se développer qu'avec l'arrivée des femmes qui sont à l'origine de l'art culinaire maghrébin (Hal, 1996 :19). Celles-ci vont immigrer massivement dans les années soixante dix par une procédure dite de « regroupement familial » après la décision de l'État français de « fermer » les frontières de l'immigration (1974).

29. Cette expression n'est nullement péjorative. Faire rassir une viande en boucherie traditionnelle c'est la faire maturer pour lui donner des qualités gustatives spécifiques.

30. Pendant le ressuage, la graisse se solidifie et contribue à augmenter la fermeté de la viande Plus ce travail de maturation dure et plus la viande est aisée à découper. Inversement lorsqu'elle est très fraîche, les fibres sont moins resserrées et le travail au couteau est plus délicat. La plupart des bouchers musulmans n'ont pas reçu de formation et maîtrisent mal l'art de la découpe. Ils travaillent donc essentiellement des avants de carcasse, plus facile à travailler desquels ont extrait les morceaux à bouillir pour es tagines, couscous et autres plats bouillis.

31. Rozin and Rozin, P. (1976) rapporté par Beardsworth and Keil (1997).

32. Pour donner un exemple tunisien le niveau de consommation de viande entre les plus pauvres et les plus riches se situe dans un rapport quantitatif de un à sept (Jaouadi, 2000).

33. Un décret du $1^{\text {er }}$ octobre 1980 stipule qu'il est interdit de procéder à un abattage rituel en dehors d'un abattoir.

34. La souffrance animale est le principal argument des associations dites de protection animale qui mènent campagne contre certaines conditions d'abattages lors de la fête de l'Aïd.

35. «Vous sont interdits la bête trouvée morte, le sang, la chair de porc, ce sur quoi on a invoqué un autre nom que celui d'Allah... » (Le Saint Coran, traduction Muhammad Hamidullah, extrait du verset 3 de la sourate 5).

36. Le développement phénoménal de ces sites Internet et notamment des forum de discussion, leur intérêt sociologique a été étudié par Gary R. Bunt (2003).

37. Deux sites francophones parmi les plus fréquentés: http://www.oumma.com - http:// www.islamie.com

38. Interdit, antonyme de halal.

39. L'absence d'intermédiaire dans les pratiques traditionnelles, où l'abattage de la volaille a souvent lieu à proximité de la cuisine, a probablement facilité son industrialisation dans le pays d'émigration dans la mesure où il n'y a pas de fonction intermédiaire entre producteur et consommateur.

40. Dans la convention industrielle, les biens sont définis par des règles objectives, dans des « cahiers des charges». 
41. On achète une volaille vivante puis on la tue dans la cuisine ou dans le jardin avant de la préparer pour la cuire.

42. Voir paragraphe sur les revenus du marché de la certification halal dans Bergeaud-Blackler (2005).

43. Le Codex Alimentarius est un code alimentaire représentant la référence mondiale pour les consommateurs, les producteurs et les transformateurs de denrées alimentaires, les organismes nationaux de contrôle des aliments et le commerce international des produits alimentaires. Entre autres, il établit les normes sur l'étiquetage des denrées alimentaires, sur les méthodes d'analyse et d'échantillonnage, sur les systèmes d'inspection et de certification des importations et exportations alimentaires etc.

44. Pour une vue d'ensemble lire le rapport « Halal Food Products Market Report » de l'International Markets Bureau - Market and Industry Services Branch sur Agriculture and Agri-Food Canada official website « Halal Food Products Market Report ».

45. Le Codex Alimentarius énonce la liste des animaux et plantes, qui ne peuvent, comme tels ou sous formes résiduelles, entrer rans la composition d'un produit étiqueté halal.

46. De plus amples investigations sont nécessaires pour savoir si la normalisation des échanges internationaux est à l'origine du développement de cette nouvelle conception et/ou si les rapports de forces internationaux ont privilégié une conception du halal particulière à une région économiquement plus dynamique dans ce secteur (comme certains pays du Sud Est asiatique).

\section{RÉSUMÉS}

Sur la base d'enquêtes sur la production de viandes bouchères halal, réalisées dans le Sud-Ouest, cet article propose de décrire et d'analyser le développement des marchés halal en France. S'appuyant sur des éléments de la théorie des conventions pour laquelle la qualification d'un produit est un processus continu tout au long de la vie de celui-ci, l'auteur brosse un tableau des principaux déterminants démographique, économiques et sociaux de l'établissement des marchés halal et de la naissance du halal business. En quelques décennies on est passé d'une conception du halal où l'acte d'égorgement revêt une importance primordiale à une conception où halal devient synonyme de pureté. Cette nouvelle définition accompagne une modification profonde de l'organisation des circuits halal qui tend peu à peu vers la spécialisation sur le mode des filières qualité. L'auteur conclue que l'entrée prévisible de la grande distribution dans ce secteur devrait s'accompagner d'une réflexion sur la définition, la présentation des produits halal et sur le droit (national et européen) à la protection et à l'information des consommateurs.

From Halal Meat to Halal Food. How Halal Markets Developed in France?

This article describes and analyzes the development of halal markets in France using results from studies carried out south-west region on the production of halal fresh meat. Based on the theory of conventions, for which the qualification of a product is a continuous process throughout its lifecycle, the author shows how, through social, economic conventions halal markets and products have evolved and have even modified the definition of halal. One passes from a design of halal where the act of slaughtering is of primary importance to a format where halal becomes synonymous with purity. This new design accompanies a profound change of the organization from halal circuits to distinct and separate channels. The predicted future entry of major 
retailers into this sector should be accompanied by a reflection on the rights of halal consumers to protection and to access to information.

De la carne halal al halal food : ¿ Cómo el comercio halal se ha desarrollado en Francia ?

A partir de una serie de encuestas sobre la producción de carnes halal en el Suroeste francés, este artículo describe y analiza el desarrollo de mercados halal en Francia. Apoyándose en la teoría de las convenciones según la cual la calificación de un producto debe ser vista como un proceso continuo que actúa a lo largo de toda su vida, el autor esboza una tabla de los determinantes demográficos, económicos y sociales con peso en el establecimiento de mercados halal y en el nacimiento del halal business. En sólo varias décadas hemos pasado de una concepción del halal en la cual el acto de degollar revierte une importancia primordial a otra concepción dónde el halal se convierte en sinónimo de pureza. Esta definición nueva rinde cuentas de cambios profundos en la organización de los circuitos halal, los cuales tienden a especializarse siguiendo el modelo de las cadenas de calidad. El autor concluye que la entrada previsible de la gran distribución en este sector debería ser acompañada por una reflexión sobre la definición, la presentación de los productos halal y el derecho (nacional y europeo) a la protección y a la información de los consumidores.

INDEX

Mots-clés : alimentation, pratique culturelle, pratique religieuse Index géographique : France

\section{AUTEUR}

\section{FLORENCE BERGEAUD-BLACKLER}

Sociologue. Chercheuse associée à l'Unité d'Anthropologie : Adaptabilité Biologique et Culturelle UMR 6578 CNRS - Université de la Méditerranée, Faculté de Médecine. 27, bd Jean Moulin 13385 Marseille cedex 05, France. Honorary Research Associate, CRIC, Faculty of Social Sciences and Law, University of Manchester (UK). fbb@aofood.org 